\title{
Nucleation Control of Oriented Titania Nanofibers
}

Dmitry Maznichenko, ${ }^{\dagger}$ Bo Tan, ${ }^{\ddagger}{ }^{*}$ Krishnan Venkatakrishnan ${ }^{\dagger}$

${ }^{\dagger}$ Department of Mechanical and Industrial Engineering, Ryerson University, Toronto, M5B 2K3, Canada

${ }^{\ddagger}$ Department of Aerospace Engineering, Ryerson University, Toronto, M5B 2K3, Canada

\section{Corresponding Author}

*tanbo@ryerson.ca

This PDF file includes:

Section S1. Illustration of Titania Nanofiber Network

Section S2. Further Explanation of Oriented Attachment of the Titania Nanofiber

References for Supplementary Material 

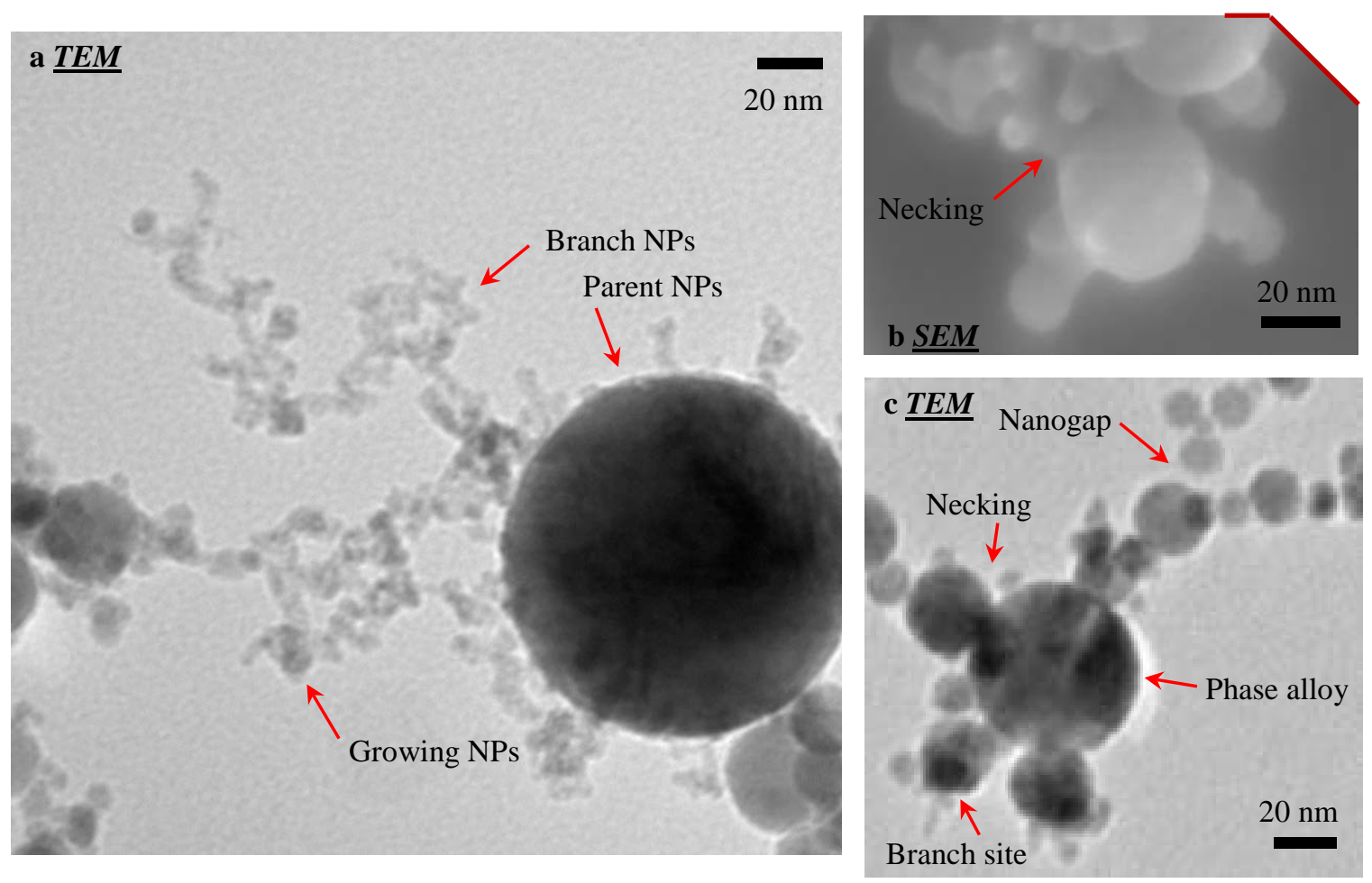

Figure S1. Titania nanofibre self-orientation, branching, and growth. Nanogap, necking and phase alloying are clearly distinguishable. (a) Nanofibre branching and growth supported by the parent NP. (b) necking, strong NP interaction. (c) nanofibre growth and phase transformation. 


\section{S2. FURTHER EXPLANATION OF ORIENTED ATTACHMENT OF THE TITANIA} NANOFIBER

Selection rules have been calculated for rutile on expected crystal growth planes. It was previously noted that the $A_{1 g}$ mode increases relatively to the $E_{g 1}$ mode. The 3-D and quasirandom orientation of the nanofibres ensures that all three modes will be detected to some capacity. In essence, this means that a local effect is prominent that is causing the relative intensity changes between the $A_{1 g}$ and the $E_{g}$ modes.

The Raman polarization tensors for the various Raman-active modes of rutile are the following: ${ }^{1}$

$$
\begin{gathered}
\Gamma_{1}\left(A_{1 g}\right)=\left(\begin{array}{lll}
a & 0 & 0 \\
0 & a & 0 \\
0 & 0 & b
\end{array}\right) \\
\Gamma_{5}\left(E_{g}, \mathrm{xz}\right)=\left(\begin{array}{lll}
0 & 0 & e \\
0 & 0 & 0 \\
e & 0 & 0
\end{array}\right) \\
\Gamma_{5}\left(E_{g}, \mathrm{yz}\right)=\left(\begin{array}{lll}
0 & 0 & 0 \\
0 & 0 & e \\
0 & e & 0
\end{array}\right)
\end{gathered}
$$

For the calculation of the relative Raman intensities, the following linear algebra operation was performed: ${ }^{2}$

$$
I_{\mu \nu} \sim\left|\left\langle\mathbf{e}_{\mathrm{i}}^{u} \cdot \boldsymbol{\Gamma} \cdot \mathbf{e}_{\mathrm{s}}^{v}\right\rangle\right|
$$

where $\mu$ and $v$ are the directions of the polarization unit vector of the incident $\left(\mathbf{e}_{\mathbf{i}}\right)$ and scattered $\left(\mathbf{e}_{\mathbf{s}}\right)$ laser beam in the considered preferential growth planes of rutile $\mathrm{TiO}_{2}$. These vectors together with the calculated polarized intensity are summarized by Table S1. 
Table S1. Selection rules for selected rutile planes.

\begin{tabular}{|c|c|c|c|c|}
\hline $\begin{array}{l}\text { Crystal } \\
\text { Plane }\end{array}$ & $\begin{array}{l}\text { Principal Axis } \\
\left(\mathbf{e}_{\mathrm{i}}, \mathbf{e}_{\mathrm{s}}\right)\end{array}$ & $\begin{array}{l}I_{\mu \nu}\left(A_{1 g}\right) \\
611 \mathrm{~cm}^{-1}\end{array}$ & $\begin{array}{l}I_{\mu \nu}\left(E_{g}, \mathrm{Xz}\right) \\
443 \mathrm{~cm}^{-1} \\
\end{array}$ & $\begin{array}{l}I_{\mu \nu}\left(E_{g}, \mathrm{yz}\right) \\
433 \mathrm{~cm}^{-1}\end{array}$ \\
\hline \multicolumn{5}{|l|}{$(100)$} \\
\hline & $\mathrm{y}, \mathrm{y}=[010],[010]$ & $a^{2}$ & 0 & 0 \\
\hline & $\mathrm{y}, \mathrm{z}=[010],[001]$ & 0 & $\mathrm{e}^{2}$ & 0 \\
\hline & $\mathrm{z}, \mathrm{z}=[001],[001]$ & $b^{2}$ & 0 & 0 \\
\hline & $x, x=[100],[100]$ & $a^{2}$ & 0 & 0 \\
\hline & $\mathrm{x}, \mathrm{y}=[100],[010]$ & 0 & 0 & 0 \\
\hline & $\mathrm{y}, \mathrm{y}=[010],[010]$ & $a^{2}$ & 0 & 0 \\
\hline & $\mathrm{x}^{\prime}, \mathrm{x}^{\prime}=[1 \overline{1} 0] / \sqrt{2},[110] / \sqrt{2}$ & $(a+b)^{2} / 4$ & $\mathrm{e}^{2}$ & 0 \\
\hline & $\mathrm{x}^{\prime}, \mathrm{y}^{\prime}=[1 \overline{1} 0] / \sqrt{2},[110]$ & 0 & 0 & $\mathrm{e}^{2} / 2$ \\
\hline & $\mathrm{y}^{\prime}, \mathrm{y}^{\prime}=[110],[110]$ & $a^{2}$ & 0 & 0 \\
\hline & $x^{\prime \prime}, x^{\prime \prime}=[10 \overline{1}] / \sqrt{2},[10 \overline{1}] / \sqrt{2}$ & $(a+b)^{2} / 4$ & 0 & 0 \\
\hline & $\mathrm{x}^{\prime \prime}, \mathrm{y}^{\prime \prime}=[10 \overline{1}] / \sqrt{2},[010]$ & 0 & 0 & 0 \\
\hline & $\mathrm{y}^{\prime \prime}, \mathrm{y}^{\prime \prime}=[010],[010]$ & $a^{2}$ & 0 & 0 \\
\hline & $\mathrm{x}^{\prime \prime \prime}, \mathrm{x}^{\prime \prime \prime}=[100],[100]$ & $a^{2}$ & 0 & 0 \\
\hline & $\mathrm{x}^{\prime \prime \prime}, \mathrm{y}^{\prime \prime \prime}=[100],[01 \overline{1}] / \sqrt{2}$ & 0 & $\mathrm{e}^{2} / 2$ & 0 \\
\hline & $\mathrm{y}^{\prime \prime \prime}, \mathrm{y}^{\prime \prime \prime}=[01 \overline{1}] / \sqrt{2},[01 \overline{1}] / \sqrt{2}$ & $(a+b)^{2} / 4$ & 0 & $\mathrm{e}^{2}$ \\
\hline
\end{tabular}

From the results of Table $\mathrm{S} 1$, it is clear that the $A_{1 g}$ mode has a high and resilient overall intensity. The intensity of the $E_{g}$ mode, however, is more dependent on the crystal orientation and on average is not expected to be as strong. The relative strength of these two modes does differ in the nanofiber network per Figure 3 of the article. For rutile, the (001) plane has the highest surface energy with a high magnitude of surface atomic relaxation. ${ }^{3}$ On the other hand, the (110) plane is the most stable and abundant with a low magnitude of surface atomic relaxation. ${ }^{4}$ The relative increase in intensity of the $A_{1 g}$ mode to the $E_{g}$ mode occurs with NP growth and orientation as nanofibers in the three-dimensional web system.

Oriented attachment (OA) of the titania NPs is clearly evident in this study. SEM scanning was performed on very low density nanofibres to investigate the concern of artificial structuring due 
to the preparation process for TEM imaging. As seen from Figure S2, the artificial structuring due to removing the nanofibres from the Ti substrate is minimal.
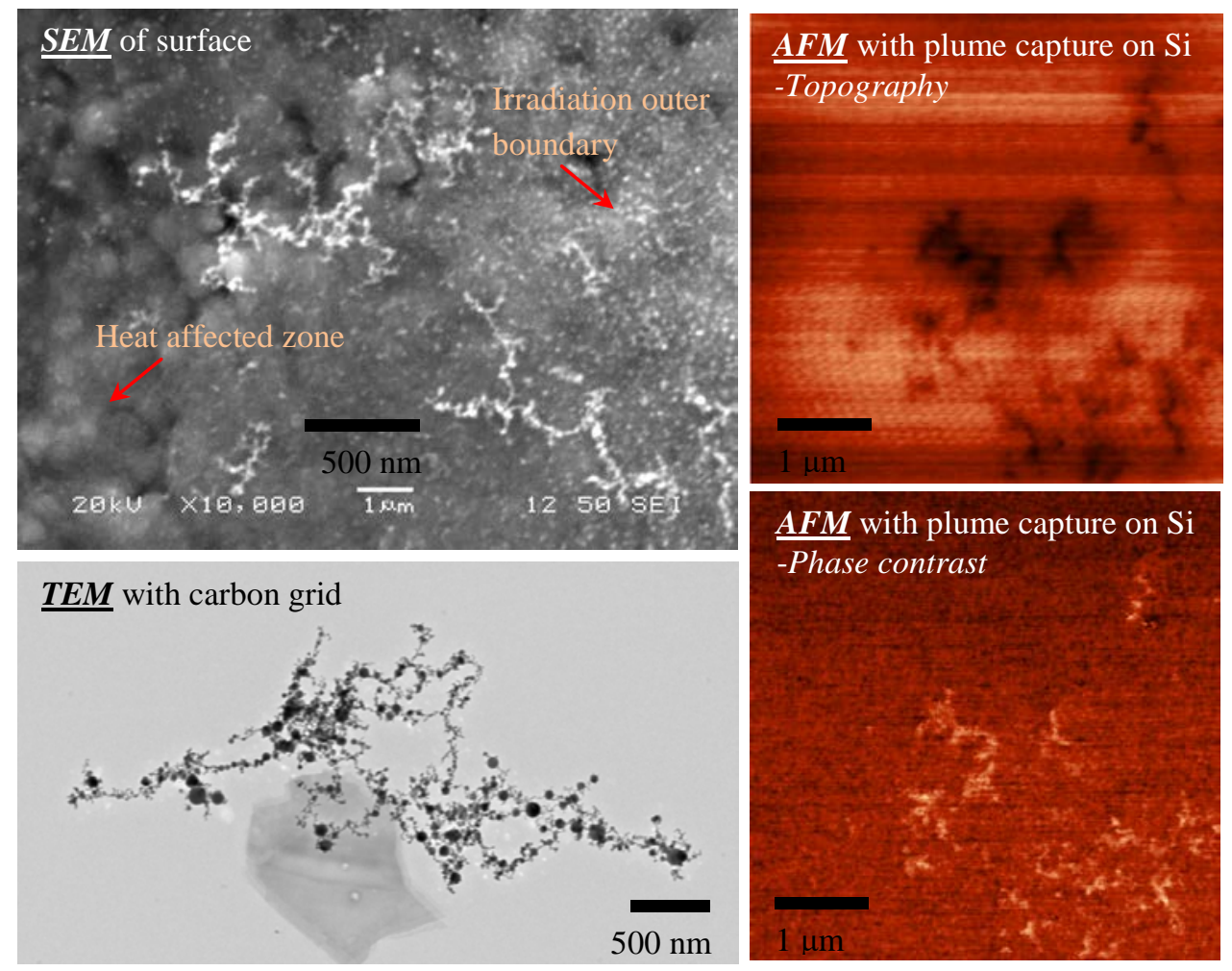

Figure S2. Transferring of titania nanofibres from the original Ti substrate (seen by SEM scanning) to the carbon grid for TEM studies. Plume species are analyzed with AFM.

From the semi-contact AFM scans of Figure S2, the particles are found to sink into the ORMOCER coated Si (during or after plume synthesis) as revealed by the negative z-direction feedback. AFM phase images confirm that the NPs are different from the Si/polymer substrate.

Contact force measurements were also performed with AFM on as-synthesized titania networks (Figure S3). Upon approach, it is observed that the nanofibrous network resists the penetrating force of the cantilever tip. Upon retraction, the nanofibres continue to adhere to the tip as they are pulled away from the substrate. In doing so, the sliding, rolling, and stretching of the aggregates prior to the final rupture is observed by signal fluctuations (0.5 - $1 \mathrm{nN}$ steps). Such observations also persist through longer cantilever movements with denser and thicker nanofibre 
networks (e.g. $13 \mathrm{MHz}$ - $25 \mathrm{~ms}$ ). Similar $\mathrm{TiO}_{2}$ nanofibres have been tested mechanically and found to have a high elasticity ( $>100 \%$ stretch), ultimate strength at ca. $2.5 \mathrm{nN}$, and resist kinetic impact up to $52 \mathrm{~m} / \mathrm{s}^{5-7}$

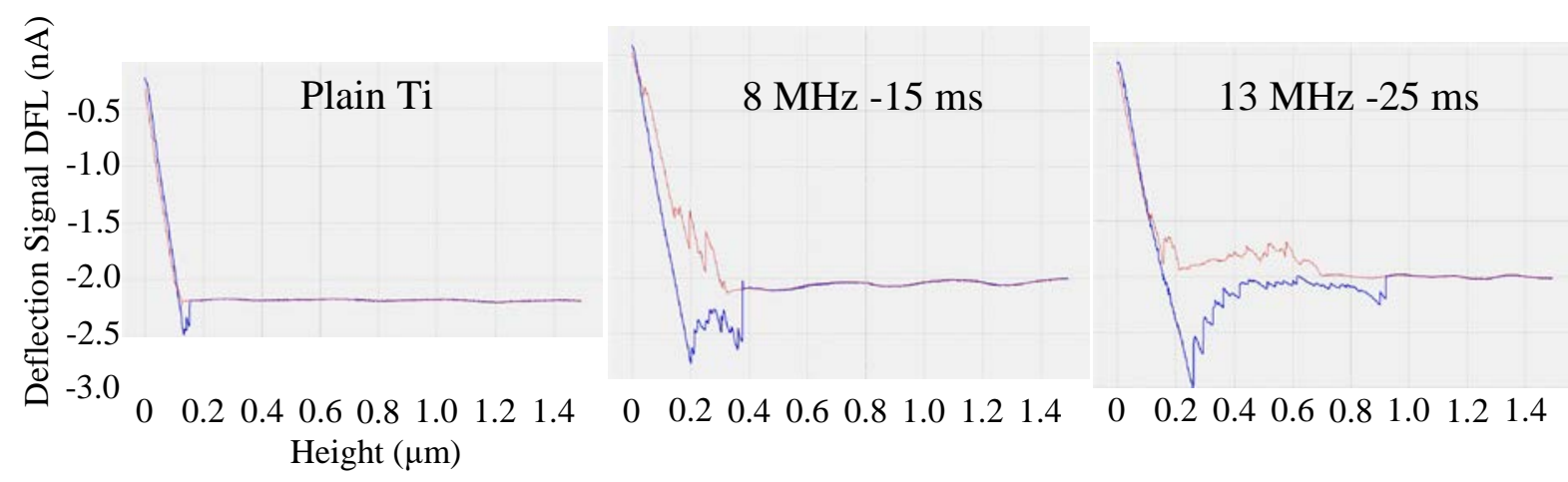

Figure S3. AFM adhesion force curves (red - on approach, blue - on retract).

The results presented with this section suggest a high level of nanoscale stability of the nanofiber network. This is due in part because of mechanical resiliency due to the atomically coordinated oriented attachment of the nanofibers. The synthesis methods allows a degree of freedom in network assembly which inherently impacts micro- and macro- material property homogeneity. Prior study has revealed a roughness parameter per scanning near-field microscopy method (SNOM) that quantified an optimum network porosity. ${ }^{8}$ The study also identified the presence of SERS hot-spots. As a result, statistical sampling methods are required to fully capture the potential of such dielectric nano-materials. ${ }^{9,10}$ 


\section{REFERENCES FOR SUPPORTING INFORMATION}

(1) Merle, P.; Pascual, J.; Camassel, J.; Mathieu, H. Uniaxial-Stress Dependence of the FirstOrder Raman Spectrum of Rutile. I. Experiments. Phys. Rev. B 1980, 21, 1617-1626.

(2) Korotcov, A. V.; Huang, Y.-S.; Tiong, K.-K.; Tsai, D.-S. Raman Scattering Characterization of Well-Aligned RuO2 and IrO2 Nanocrystals. J. Raman Spectrosc. 2007, 38, 737-749.

(3) Labat, F.; Baranek, P.; Adamo, C. Structural and Electronic Properties of Selected Rutile and Anatase TiO2 Surfaces: An Ab Initio Investigation. J. Chem. Theory Comput. 2008, 4, 341352.

(4) Deskins, N. A.; Kerisit, S.; Rosso, K. M.; Dupuis, M. Molecular Dynamics Characterization of Rutile-Anatase Interfaces. J. Phys. Chem. C 2007, 111, 9290-9298.

(5) Suh, Y. J.; Friedlander, S. K. Origins of the Elastic Behavior of Nanoparticle Chain Aggregates: Measurements Using Nanostructure Manipulation Device. J. Appl. Phys. 2003, 93, 3515-3523.

(6) Salameh, S.; Schneider, J.; Laube, J.; Alessandrini, A.; Facci, P.; Seo, J. W.; Ciacchi, L. C.; Mädler, L. Adhesion Mechanisms of the Contact Interface of TiO 2 Nanoparticles in Films and Aggregates. Langmuir 2012, 28, 11457-11464.

(7) Seipenbusch, M.; Froeschke, S.; Weber, A. P.; Kasper, G. Investigations on the Fracturing of Nanoparticle Agglomerates-First Results. Proc. Inst. Mech. Eng. Part E J. Process Mech. Eng. 2002, 216, 219-226.

(8) Maznichenko, D.; Selvaganapathy P.R.; Venkatakrishnan, K.; Tan, B. TiO2 Nanofibrous Interface Development for Raman Detection of Environmental Pollutants. Appl. Phys. Lett. 2012, 101, 231602. 
(9) Alessandri, I.; Lombardi, J. R. Enhanced Raman Scattering with Dielectrics. Chem. Rev. 2016, $116,14921-14981$.

(10) Alessandri, I.; Vassalini, I.; Bertuzzi, M.; Bontempi, N.; Memo, M.; Gianoncelli, A. "RaMassays": Synergistic Enhancement of Plasmon-Free Raman Scattering and Mass Spectrometry for Multimodal Analysis of Small Molecules. Sci. Rep. 2016, 6, 1-8. 